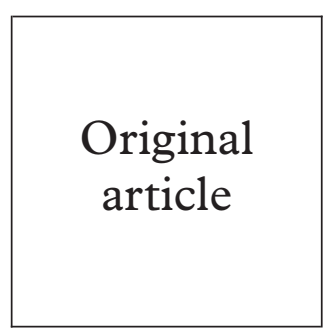

\title{
Integrated clinical service for sexual assault victims in a genitourinary setting
}

\author{
C P E H Bottomley, T Sadler, J Welch
}

Background: Reported sexual assault is increasing, and the diverse immediate and longer term needs of the victim are usually met by exposure to a number of healthcare professionals often in different locations, involving delays and travel, increasing the trauma for the victim.

Objectives: To set up a centre to address the immediate and longer term needs of the sexual assault victim and review issues arising during the development of the service.

Methods: Description of setting up the service in the genitourinary medicine department of Kings College Hospital, south London, and the aspects of care offered.

Results: The number of victims referred by police increased from 15 in 1992 to 58 in 1996. In 1996, 55 female and three male victims were seen. 23 different police stations brought victims for examination; mean age of the victim was 27 years (range 14-60), median time between assault and examination was 22 hours (range 3 hours-3 months); $23 \%$ had genital injuries, 59\% had other physical injury, and $11 \%$ needed further hospital care. $71 \%$ accepted screening for sexually transmitted infection (STI), $21 \%$ had an STI diagnosed, $16 \%$ of the women required emergency contraception, $26 \%$ received prophylactic antibiotics, and $58 \%$ saw a health adviser. $70 \%$ had a follow up appointment arranged of which $50 \%$ attended.

Conclusion: The high uptake of STI screening, emergency contraception, health adviser consultation, and follow up supports the concept of a comprehensive integrated system to meet the disparate needs of the victim while still obtaining the necessary forensic evidence. The wide catchment area of service users indicates gaps in services available for the assault victim. Earlier genitourinary involvement after sexual assault is becoming increasingly pertinent in relation to HIV prophylaxis.

(Sex Transm Inf 1999;75:116-119)

Keywords: sexual assault; rape; STIs; HIV

\section{Introduction}

Reported sexual assault is increasing, with 7708 allegations of sexual assault and 1750 allegations of rape made to the Metropolitan Police in the year 1996/1997. ${ }^{1}$ Many victims do not report to the police and others are traumatised by their treatment after reporting and this leads to fewer successful prosecutions. ${ }^{2}$ Internationally, different approaches are taken to the collection of forensic evidence and to the clinical and psychological treatment of alleged victims..$^{3-5}$ For example, in some US states nurse led teams perform forensic genital examinations, collect evidence for analysis, and arrange counselling and psychological follow up. In the United Kingdom practice varies according to police region. For example in Manchester, St Mary's Sexual Assault Centre offers forensic examination and counselling linked with an appointment for sexually transmitted infection (STI) investigation and advice with a local genitourinary medicine (GUM) physician within 2 weeks. ${ }^{6}$

Traditionally, within the Metropolitan Police area victims have been offered forensic examination in a victim examination suite (generally annexed to a police station or within a hospital) and then advised on where to receive appropriate GUM, contraception, and counselling follow up. Specific problems in London have been the shortage of facilities for thorough genital examination, delays between requests for examination, and an appropriately trained forensic examiner being available, and difficulty in upholding the Home Office guideline that a victim has the right to an examiner of the same sex. ${ }^{7}$

The Camberwell Clinic at King's College Hospital was set up to address these issues. It provides a reservoir of trained male and female forensic examiners and healthcare professionals as well as liaison with accident and emergency staff when necessary, and aims to encompass on one site the acute and ongoing needs of male and female sexual assault victims. These needs include forensic examination and evidence collection, treatment of physical injuries, consideration of emergency contraception, psychological support, investigation and treatment for sexually transmitted infections (STI) (including HIV and hepatitis), and subsequent follow up as necessary.

The development and practice of this integrated service are described with a review of the issues arising as it has expanded. (The term victim is used throughout this article to mean a man or woman who has reported an incident of sexual violence against them. No conclusions are drawn as to the truth of the accusation.)

\section{Methods}

In 1992 the Department of Sexual Health at King's College Hospital was asked by the Metropolitan Police to provide a forensic service for sexual assault victims to be integrated with 
the provision for their psychological and medical needs. Initially a core group of doctors from the department attended the Metropolitan Police training programme on forensic examination following alleged sexual assault and offered a forensic service at the clinic on an ad hoc basis. This has now expanded to 3 days each week during normal clinic opening hours, when forensic examinations can be performed with support from nurses and health advisers who have also attended the training course. A clinical research fellow has been introduced to see the majority of the victims and develop the service, coordinating within the department and liaising with both local police stations and representatives from the Forensic Medical Services Branch of the Metropolitan Police.

Most victims of sexual assault are referred for examination by the Metropolitan Police, but some present directly to the clinic. In the latter case staff offer to contact the police on their behalf but if this is declined then forensic examination can be carried out and any specimens taken stored in a locked freezer while the victims consider whether they wish to report the incident later. All requests for forensic examination are referred by reception staff to the senior nurse or doctor on forensic duty who clarifies details. Police chaperons escorting victims are asked to estimate time of arrival, wear plain clothes, and state their attendance for "Camberwell Clinic" on arrival so as not to draw unnecessary attention to the victim. Victims are seen immediately in a designated room in which special equipment, including disposable specula and proctoscopes, is available. A prepacked "forensic kit" containing swabs for evidence collection, specimen bottles for blood, urine, and saliva samples, self sealing labelled specimen bags, and anatomical line drawings for recording findings is brought by the police chaperon.

There are currently six clinic doctors (two male and four female, from senior house officer to consultant grade) trained in forensic examination of the sexual assault victim. The doctor may be assisted either by the police chaperon or by one of the 10 clinic nurses who have also received formal training. The latter assists if the victim declines the presence of the police chaperon at examination or if the victim has yet to decide whether to report the assault to the police. A health adviser (with specific counselling training) is always available to address the psychological needs of the victim after completion of the physical examination.

Forensic examination takes place as soon as possible after the assault and though sampling for the assailant's DNA may be futile after 7 days there is sometimes benefit in performing an examination weeks or months later if persisting injuries are suspected.

The doctor takes a brief history of the assault from the police chaperon (often in a separate room but then confirmed with the victim) in order to direct the examination and reduce repetition for the victim. The doctor then explains the procedure, emphasising the difference between a forensic examination, which is for legal purposes with the likelihood of findings being heard publicly in the courtroom, and a normal confidential doctor-patient consultation. The doctor obtains written consent from the victim (and from an appropriate adult if, for example, there are mental health issues) to take a history, perform an examination, collect samples, record findings in personal notes, write a statement, and give evidence in court if required.

\section{FORENSIC EXAMINATION}

A full head to toe examination is performed and any injuries are recorded and sketched onto standard anatomical line drawings with measurements of size and distance from bony landmarks. Genital examination with speculum, anorectal examination with proctoscope, and bimanual pelvic examination are carried out as directed by the history. Relevant material (such as vaginal swabs, anal swabs, blood, and urine) is taken for evidence, specimens being labelled, sealed, signed, and checked to police standards. The method of forensic examination is fully described in other publications. ${ }^{8}$

SEXUALLY TRANSMITTED INFECTIONS

The risk of STI, including HIV and hepatitis, is discussed with every victim. STI screening is advised at 3-7 days and at 2 weeks after the assault (as dictated by incubation periods of Neisseria gonorrhoeae and Chlamydia trachomatis) or later if prophylactic antibiotics have been taken. Therefore, samples are only taken at the time of forensic examination if the assault was more than 3 days earlier, the victim has been recurrently raped over a period of time, or is deemed unlikely to attend follow up. In such cases the examining doctor or the nurse takes the samples (following standard clinic procedures) and the nurse then carries out microscopy. Any immediate results (and treatment if necessary) are given by the nurse or another clinic doctor, without the examining doctor or police chaperon present. Thus the doctor carrying out forensic examination and the police are not aware of any STI present which could adversely influence the legal case.

Syphilis testing is performed and, after obtaining verbal consent, a sample of serum is stored indefinitely in the virology department for comparison should a future sample suggest HIV, hepatitis, or syphilis infection and an attempt be made to date the exposure to the virus.

Although HIV is discussed at the initial consultation, antiretroviral prophylaxis has only been given in a few selected recent cases. It has been suggested that postexposure prophylaxis against HIV should be offered to rape victims,${ }^{9} 10$ and victims at the Camberwell Clinic are assessed individually and potential risks of HIV infection balanced against the side effects, anxiety, and expense of prophylaxis.

Prophylactic antibiotics against $C$ trachomatis, $N$ gonorrhoeae, and Trichomonas vaginalis are offered to the victim (especially if there were multiple or unknown assailants), the regimen prescribed being taken from the department prescribing protocol (similar antimicrobial cover as for suspected pelvic inflammatory dis- 
ease). Hepatitis B immunoglobulin and accelerated vaccination are considered as well as HIV prophylaxis. Serum testing is suggested at 3 months (for HIV and syphilis) and 6 months (for hepatitis) depending on the details of the assault and the patient's anxiety.

Microbiology samples from sexual assault victims are labelled "medicolegal" and handled individually (as opposed to being integrated with the routine clinic specimen processing). Positive isolates are stored and further investigations arranged as necessary, such as typing of $N$ gonorrhoeae. However, after consideration within the clinic and with Metropolitan Police Forensic Medical Branch it is generally only deemed relevant to attempt to use such evidence to support the forensic case in victims at the extremes of age.

\section{CONTRACEPTION}

Both hormonal postcoital contraception and insertion of an intrauterine contraceptive device are available. Counselling is available if the woman is already pregnant and referral for termination can be expedited.

\section{PSYCHOLOGICAL SUPPORT}

After the examination the victim is offered a shower and a drink, while the medical notes are completed and the police chaperon finishes the labelling and sealing of specimens. The doctor then explains the findings to the victim who is encouraged to see a health adviser at that time.

The health adviser usually spends 10-30 minutes alone with the victim, explaining the common psychological reactions to the trauma of sexual assault and the available support services. Some victims opt for several counselling sessions with the health adviser who saw them at their initial attendance, while others decline further counselling or are referred to more specialised agencies.

FOLLOW UP

Follow up appointments (at the Camberwell Clinic or a clinic local to the victim's home) are arranged for 3-7 days, 2 weeks, and 3 months from the time of the assault. A doctor who did not conduct the forensic examination sees the victim on subsequent attendances to avoid a conflict of interest for the forensic doctor.

The doctor keeps contemporaneous notes of the forensic examination using a proforma. Verbatim detailing of history is avoided as minor differences between the doctor's notes and the formal police statement may be highlighted in court and jeopardise the legal case. Line drawings are carefully completed, annotated and labelled, copies being given to the police with the specimens while the doctor keeps the original. Confidential clinic notes are completed separately to be referred to when the victim returns for follow up for STI, pregnancy concerns, or psychological issues.

UPTAKE OF SERVICES

Each year an increasing number of victims have been referred for examination and in 1996 the 58 victims seen were referred from 23 different London police stations. During that year the mean age of victim was 27 years (range 14-60) and median time from assault to examination was 22 hours (range 3 hours to 3 months). Genital injuries were found in $23 \%$ and other physical injuries in $59 \%$, with $11 \%$ requiring further hospital care.

Screening for STI was accepted by $71 \%$ of the victims of whom $21 \%$ were diagnosed with one or more infection (three $C$ trachomatis, two $N$ gonorrhoeae, one Molluscum contagiosum, one Trichomonas vaginalis, one hepatitis B and C).

Prophylactic antibiotics were prescribed for $26 \%$, and emergency contraception was required by $16 \%$ of the women: $58 \%$ saw a health adviser and $70 \%$ had a follow up appointment made at the Camberwell Clinic, of which 50\% attended.

FUNDING

Funding is provided by Metropolitan Police Forensic Medical Services paying the clinic a modest retainer fee for each "on call" day, plus a standard fee per examination. The examining doctor is paid for statements and court appearances.

\section{Discussion}

The Camberwell Clinic was the first centre in the United Kingdom to offer a comprehensive forensic and follow up service to victims of sexual assault in a genitourinary setting. Not only does the service reduce the time involved and potential number of health professionals likely to be encountered but the open access (walk in) follow up system offers advantages for those victims whose circumstances create difficulty in keeping appointments.

Metropolitan Police chaperons and investigating officers give positive feedback on the forensic and follow up service offered by the Camberwell Clinic and meetings between clinic staff and police aid cooperation in providing for victims.

\section{PROBLEMS}

The number of sexual assault victims examined at the Camberwell Clinic has greatly increased over the 5 years since its inauguration. This has put strain on the general clinic space and workload as well as on the individual doctors involved. A new examination suite with adjacent shower room within the clinic has been provided which relieves the inconvenience of a general GUM consultation room being occupied, as well as providing a more informal, less clinical environment for the victim.

Staff turnover is high so training is an ongoing issue. New doctors joining the clinic are skilled in general genital examination but initially lack forensic experience.

Statements take time to write (usually 2-3 hours) and if the case is heard in court the examining doctor may be unavailable for GUM work (and forensic examinations) for an unpredictable time and often at short notice.

There can be a conflict between gathering forensic evidence and providing medical care. The forensic doctor must take care not to elicit information such as previous sexual and infection history, which is not relevant to the foren- 
sic case but may be recalled in court, prejudicing the prosecution. The victim may therefore need to be seen by more than one doctor if treatment is required or positive microbiological results are to be given. As yet there have been no incidences where the confidential clinic notes have been requested by a judge in addition to the doctors forensic notes, though this is a potential drawback of a set of confidential clinic notes also being kept from the initial examination.

Funding for the service in the GUM setting is the same as if an individual doctor were performing the examination in a police examination suite yet it uses space and staff (health adviser, nurse, and doctor) far in excess of the reimbursement received.

\section{DEVELOPMENTS}

Since its inauguration the service has evolved in response to problems highlighted and increasing use. Examples of new developments include the dedicated examination and shower rooms, specially produced information leaflets for victims, regular liaison meetings with local police, and a more detailed protocol for the examination and follow up of the victim. Annual audit by the clinical research fellow also highlights areas for change.

The Camberwell Clinic aims to increase the referral rate of victims from south east London, while encouraging the development of similar innovative services in the other three London quadrants to provide locally for victims throughout the Metropolitan Police district.

In the 1980s it was proposed that the lack of sympathetic treatment by Metropolitan Police staff led to many victims not reporting assaults or pursuing prosecutions and, in response, specialist training was arranged by the force for officers of the sex requested to act as chaperon to the victim from the time of initial reporting of the offence through to the court case. ${ }^{2}$ The Camberwell Clinic aims to complement the improved police handling of sexual assault victims. Further innovative schemes such as this may facilitate increased reporting of sexual violence and thus help to improve the rate of prosecution of assailants.

\section{Conclusion}

Although only a minority of sexual assault allegations result in court convictions, appropriate medical care is vitally important for the physical and psychological wellbeing of victims after the alleged trauma.

With the increase in awareness of HIV and the recommendation that antiretroviral prophylaxis needs to be commenced early to be effective, consultation with a doctor in a genitourinary department is a priority for many sexual assault victims. ${ }^{9}$ We believe that a "one stop" sexual assault centre in a genitourinary setting offers the optimum integrated service for the disparate needs of victims, without compromising the collection of forensic evidence necessary for the successful prosecution of assailants. Should sexual assault victims go anywhere else?

We thank Dr Frances Lewington for her advice in the writing of this paper.

Contributors: JW formulated the idea for the paper, collected some data, and reviewed the manuscript. TS collected the majority of data. CB analysed the data. CB and TS wrote the manuscript. CB revised the manuscript after review.

1 Metropolitan Police Statistics. London: Performance Information Bureau, 1987.

2 Wagstaff, TM. New initiatives by the Metropolitan Police in the investigation of rape. Med Law 1989;8:493-8.

3 Solola A, Scott C, Severs H, et al. Rape: management in a noninstitutional setting. Obstet Gynecol 1983;61:373-8.

4 Duddle $M$. The need for sexual assault centres in the United Kingdom. BMF 1985;290:771-3.

5 Hampton $\mathrm{H}$. Care of the woman who has been raped. $N$ Engl F Med 1995;332:234-7.

6 Lacey HB. Sexually transmitted diseases and rape: the experience of a sexual assault centre. Int $\mathcal{f} S T D$ AIDS 1990;1:405-9.

7 Home Office. Violence against women. (Circular 69/86) London: HMSO, 1986

8 Courtney G, Crowe G, Forster G. Rape: examination of the victim. Medicine 1996;24:36-9.

9 Katz MH, Gerberding JL. Postexposure treatment of people exposed to the human immunodeficiency virus through sexual contact or injection-drug use. $N$ Engl $f$ Med 1997;336:1097-100.

10 Easterbrook P, Ippolito G. Prophylaxis after occupational exposure to HIV. BMF 1997;315:557-8. 